\title{
Don't swallow the NETs
}

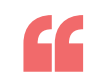

... internalization of

NETs by [fibroblast-like synoviocytes] can trigger pathogenic adaptive immunity and tissue damage

Fibroblast-like synoviocytes (FLS) internalize neutrophil extracellular traps (NETs) and present arthritogenic peptides to $\mathrm{T}$ cells, leading to autoimmunity and cartilage damage. These findings comes from a study by Carmona-Rivera et al. now published in Science Immunology. "We found that FLS from patients with rheumatoid arthritis (RA) have the capacity to internalize NETs and that this is the mechanism by which these structures activate a pro-inflammatory phenotype in FLS," says Mariana Kaplan, lead author of the study. "We also found that administration of FLS loaded with NETs to transgenic mice led to the development of anti-citrullinated protein antibody responses and cartilage damage," Kaplan continues.

Previous work has indicated that NETs are an important source of citrullinated autoantigens, which are implicated in RA pathogenesis, and that these extracellular structures are able to activate a pro-inflammatory and pathogenic phenotype in FLS. To investigate the mechanisms involved in NET-FLS interactions,

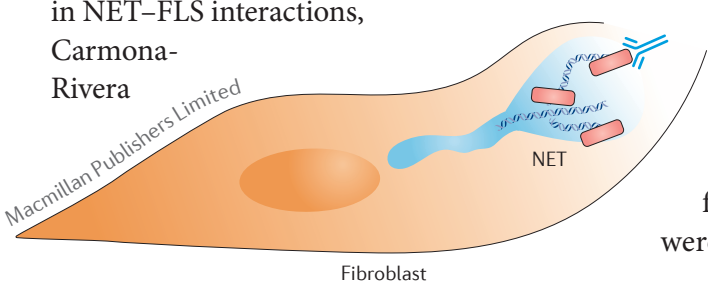

and colleagues incubated FLS derived from patients with osteoarthritis (OA) and RA, as well as control dermal fibroblasts from healthy individuals, with NETs spontaneously generated from peripheral blood neutrophils from patients with RA. By use of confocal microscopy, the investigators found that FLS from patients with OA or RA were able to internalize NETs, whereas control dermal fibroblasts showed negligible NET internalization. Furthermore, dermal fibroblasts from patients with psoriasis were also able to internalize NETs, indicating that the activation status is important in mediating this process.

Carmona-Rivera and colleagues found that NET internalization by FLS was mediated by endocytosis and regulated by the RAGE-TLR9 signalling pathway. The researchers next investigated whether NET internalization induced antigenpresenting cell properties in FLS. In FLS from patients with OA or RA, MHC class II molecules were upregulated upon exposure to NETs, a process mediated by IL-17B. "We also found that citrullinated peptides deriving from internalized NETs were presented by FLS to antigen-specific $\mathrm{T}$ cells isolated from patients with RA," explains Kaplan.

To confirm the induction of adaptive immune responses by NETloaded FLS in vivo, the investigators used a humanized $H L A-D R B 1^{*} 0401$ transgenic mouse model; such mice show susceptibility to inflammatory arthritis. Levels of anti-cyclic citrullinated peptide antibodies were higher in the sera of transgenic mice that received intra-articular injections of FLS loaded with NETs than in sera from transgenic mice injected with FLS alone, an effect that was dependent on $\mathrm{CD}^{+} \mathrm{T}$ cells. Those mice receiving intra-articular injections of NET-loaded FLS also showed reduced cartilage integrity and increased cartilage loss compared with mice injected with FLS alone.

These findings indicate that internalization of NETs by FLS can trigger pathogenic adaptive immunity and tissue damage. "The identification of this new pathway may lead to the development of new therapeutic strategies in this disease," concludes Kaplan.

Dario Ummarino

ORIGINAL ARTICLE Carmona-Rivera, C. et al. Synovial fibroblast-neutrophil interactions promote pathogenic adaptive immunity in rheumatoid arthritis. Sci. Immunol. http://dx.doi. org/10.1126/sciimmunol.aag3358 (2017) 